\title{
Quantification of Ki67 Staining Density in Tumors of the Colon Using Automated Slide Scanning Followed by Analysis Using the DensitoQuant Module in Quant Center
}

\author{
Hillary Guzik $^{1}$, Radhashree Maitra ${ }^{2}$ and Vera DesMarais ${ }^{1}$
}

${ }^{1}$ Einstein College of Medicine, Analytical Imaging Facility, Bronx, New York, United States, ${ }^{2}$ Yeshiva University and Albert Einstein College of Medicine, Bronx, New York, United States

It is very common in the study of cancer to use a large variety of tissue markers to distinguish cancer cells from healthy tissue. One commonly used marker is $\mathrm{Ki} 67$, which is clinically used to be predictive and prognostic of cancer, in diagnosis, treatment, and therapy [1]. The goal of this study was to develop a method to measure expression levels of the cell proliferation marker Ki67 in an unbiased and automated manor in tumors of the colon. Previously, pathologists have used a manual, visual scoring method to determine the amount of Ki67 in a sample. With the aid of a microscope, samples were viewed and assigned a score ranging from negative,,,++++++ based on estimates of the amount of brown color in the tissue. Such manual evaluations lead to inherent variability and bias because there are operator dependent differences in the scoring and the scoring is based on estimates, not measurements. An automated evaluation of tissue for Ki67 will improve the overall assessment, reproducibility and standardization of evaluating the Ki67 stain.

In our study, a biopsy of tumors of the colon was taken, the tissue was fixed and placed in a tissue microarray (TMA), stained for immunohistochemical (IHC) Ki67 antigen, and mounted on a standard 3 inch by 1inch slide with a \#1.5 coverslip [2]. Ki67 is a nuclear marker present throughout the active cell cycle, which provides a reliable means of evaluating cell proliferation [3]. A Ki67 positive nucleus will present itself as a brown color where a Ki67 negative nucleus will present itself in a blue color. The proliferation score will be based on the brown distribution. Slides were then scanned digitally using an automated 3D HISTECH P250 high capacity slide scanner. The advantage of using an automated slide scanner to acquire a digital image is that it has the ability to scan the whole TMA slide in minutes. Images are automatically acquired across the whole slide and stitched together to give one final high-resolution image of the whole array. This instrument can focus automatically, and can scan up to 250 slides automatically in one batch. The final image is a digital archive of the whole slide which can then be viewed or analyzed with appropriate software.

After scanning, analysis was conducted using the DensitoQuant module in Quant Center software. This analysis module detects the amount of brown stain on a pixel by pixel basis. The user can either analyze the whole piece of tissue, or the user can choose discreet regions of interest (ROIs) of variable or fixed size. Multiple ROIs can be analyzed automatically with an identical protocol, which is important for repeatability. In our study, ROIs of identical size were chosen $\left(7 \mathrm{~mm}^{2}\right)$, and thresholds were selected to distinguish the background (blue 0.99) and positive brown stain (1.04). In this protocol, the darkness of the brown stain is divided into subclasses of light, medium, and dark. These categories are flexible and are adjusted by the user at the beginning of the analysis to make sure that the selections/cut offs between categories represent the underlying biology. Both a positive and a negative sample are used to determine the settings for these categories. In our study, we used the following settings: light brown pseudo-colored in yellow a value set between 5.82 and 16.60 contains the least amount of brown, medium brown pseudocolored in orange a value set between 16.61 and 37.31 is the next densest brown, and dark brown pseudocolored in red a value set above 37.32 is the darkest/most dense brown (Figure 1). Once we established 
the protocol with control slides, the same protocol was then loaded and implemented on each slide and its respective ROIs. This generated values of the overall amount of brown in each separate ROI. The output is in either proportion percentage or total number of pixels of each category, and can be exported to Excel (Figure 2). As long as the same protocol is used, all values are reproducible, independent of the operator, allowing for an automated, quick, and unbiased analysis Ki67 expression [4].
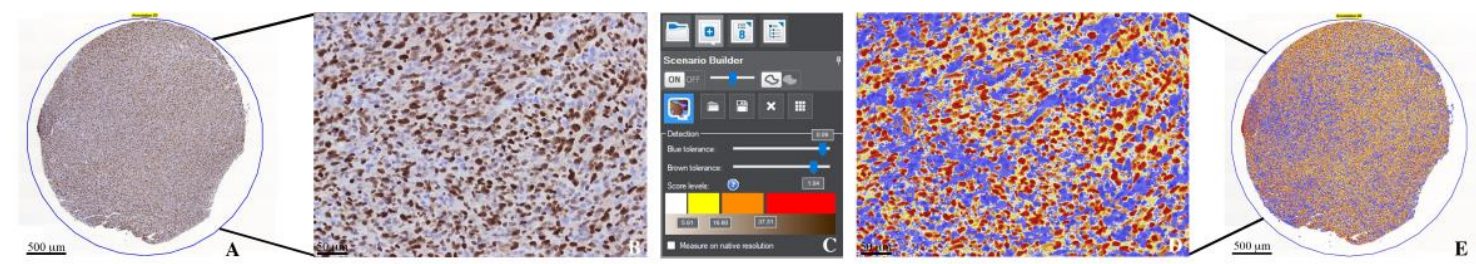

Figure 1. The analysis workflow using DensitoQuant module in Quant Center. (A) ROI selection of the biopsy of tumors of the colon stained with Ki67 at a 2.28x zoom. ROIs $(7 \mathrm{~mm} 2)$ of identical size were chosen for each TMA. (B) A 20x zoom of the Ki67 biopsy. (C) The DensitoQuant software settings are adjustable to make sure that the appropriate settings for the sample are implemented. The following settings were used: blue tolerance of 0.99 which represents the background, and brown tolerance 1.04 which represents the brown stain. The brown score level chosen are; yellow between 5.82 and 16.60 corresponding to the lightest brown, orange between 16.61 and 37.31 corresponding to the medium brown, and red above 37.32 corresponding to the dark brown. (D) The selection based on the parameters set in the DensitoQuant software. Dark brown, pseudo-colored in red is the darkest/most dense brown, medium brown pseudo-colored in orange is the next densest brown, light brown pseudo-colored in yellow contains the least amount of brown, and background pseudo-colored in blue. (E) The analysis is done on the whole ROI. ROI selection of the TMA pseudo-colored and shown at a $2.28 \mathrm{x}$ zoom. 


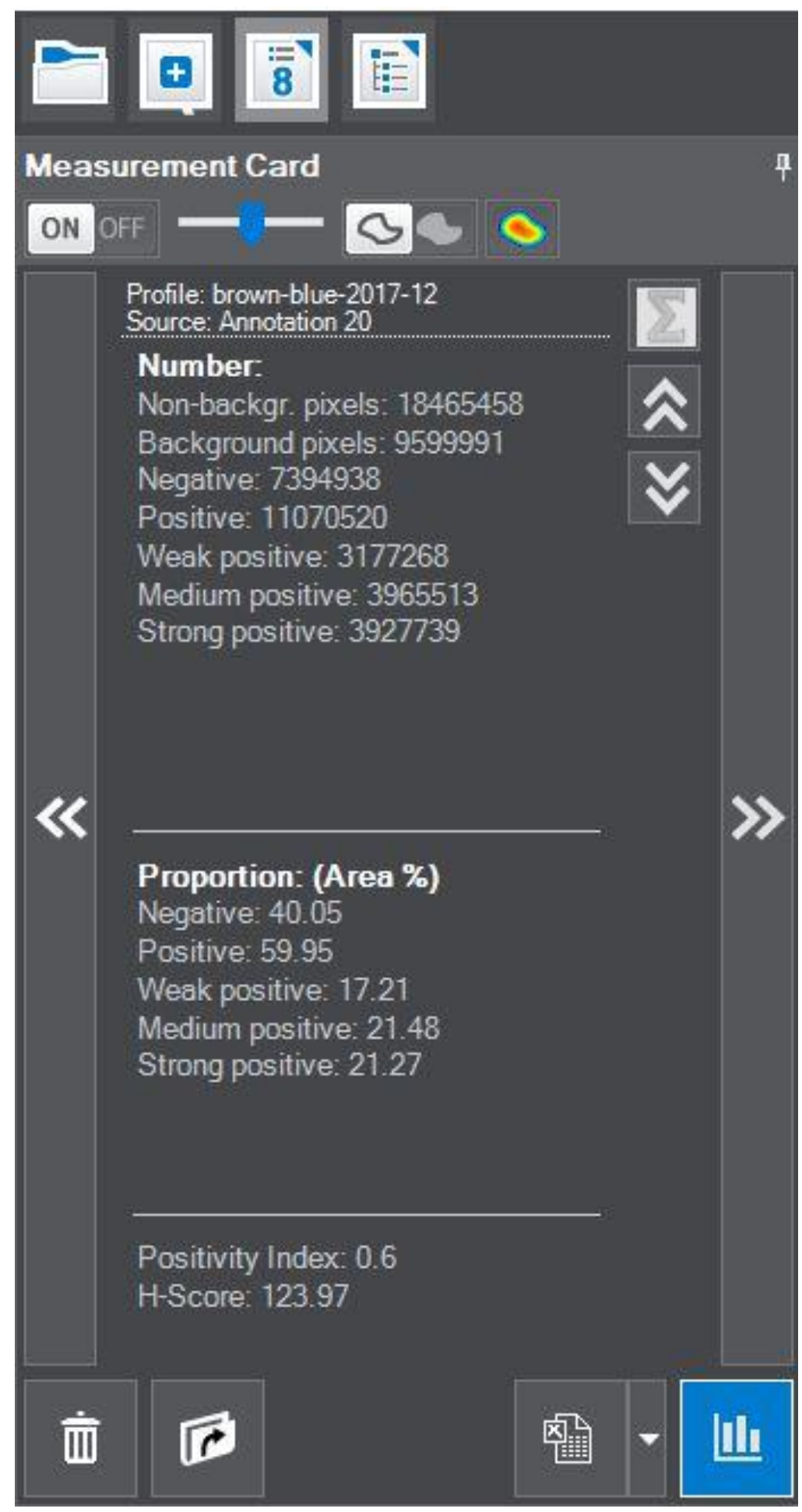

Figure 2. The analysis is done on a particular portion or on the whole ROI. We did analysis on the whole ROI. The analysis output is reported as a proportion of the area or the total amount of pixels, for each category. The proportion data for the ROI shown in Figure 1 is; Negative, the blue stain at $40.05 \%$, Positive, including all of the browns levels at 59.96\%. Also, data is given for each subclass of brown; weak positive (yellow) $17.21 \%$, medium positive (orange) $21.48 \%$, and strong positive (red) $21.27 \%$. This data can be exported into Excel. 


\section{References}

[1] Leung, S., Nielsen, T., Zabaglo, L. et al. Analytical validation of a standardized scoring protocol for Ki67: phase 3 of an international multicenter collaboration. npj Breast Cancer 2, 16014 (2016). https://doi.org/10.1038/npjbcancer.2016.14

[2] Augustine, T., Maitra, R., Zhang, J. et al. Sensitization of colorectal cancer to irinotecan therapy by PARP inhibitor rucaparib. Invest New Drugs, (2019). 37: 948. https://doi.org/10.1007/s 10637-018-007179

[3] Brown, D.C.. and Gatter, K.C.. Ki67 protein: the immaculate deception?. Histopathology, 40: 2-11 (2002). https://doi:10.1046/j.1365-2559.2002.01343.x

[4] All imaging was conducted in the Analytical Imaging Facility (AIF) (funded by NCI Cancer Grant P30CA013330). Imaging was conducted on a HISTECH P250 High Capacity Slide Scanner funded by SIG (\#1S10OD019961-01). The authors would also like to thank Frank Macaluso for proofreading the manuscript. 